OPEN ACCESS

Edited by:

Spyridon Ntougias,

Democritus University of Thrace,

Greece

Reviewed by: Ahmed Ali Saleh,

Kafrelsheikh University, Egypt

Robert Gordan Nichols, Pennsylvania State University (PSU),

United States

*Correspondence: Guang-hai Q

qiguanghai@caas.cn

Specialty section: This article was submitted to

Systems Microbiology,

a section of the journal

Frontiers in Microbiology

Received: 15 December 2019 Accepted: 18 March 2020

Published: 15 April 2020

Citation:

Wang $W-w$, Wang J, Zhang $H-j$,

Wu S-g and Qi G-h (2020)

Supplemental Clostridium butyricum Modulates Lipid Metabolism Through Shaping Gut Microbiota and Bile Acid

Profile of Aged Laying Hens.

Front. Microbiol. 11:600.

doi: 10.3389/fmich.2020.00600

\section{Supplemental Clostridium butyricum Modulates Lipid Metabolism Through Shaping Gut Microbiota and Bile Acid Profile of Aged Laying Hens}

\author{
Wei-wei Wang, Jing Wang, Hai-jun Zhang, Shu-geng Wu and Guang-hai Qi* \\ Risk Assessment Laboratory of Feed Derived Factors to Animal Product Quality Safety of Ministry of Agriculture \& Rural \\ Affairs, and National Engineering Research Center of Biological Feed, Feed Research Institute, Chinese Academy \\ of Agricultural Sciences, Beijing, China
}

Probiotic Clostridium butyricum could affect lipid metabolism in broilers. However, it is not clear whether $C$. butyricum could improve lipid metabolism through shaping gut microbiota and bile acid (BA) profile of laying hens. We aimed to evaluate the contributions of gut microbiota and BA profile to the potential effect of $C$. butyricum on lipid metabolism of aged laying hens. A total of 192 60-week-old Hy-Line Brown laying hens were divided into two groups (eight replicates per group). Birds were fed a basal diet supplemented with 0 or $2.7 \mathrm{~g} / \mathrm{kg}$ C. butyricum $\left(1.0 \times 10^{9} \mathrm{CFU} / \mathrm{g}\right)$. Samples were collected at the end of week 8 of the experiment. The results showed elevated $(P<0.05)$ concentrations of glucagon-like peptide 1 , insulin and thyroid hormones in serum responded to $C$. butyricum addition, which also decreased $(P<0.05)$ hepatic free fatty acids contents, as well as increased $(P<0.05)$ the expression of hepatic acyl-CoA oxidase, farnesoid X receptor (FXR) and PPAR $\alpha$. C. butyricum addition increased $(P<0.05)$ Bacteroidetes abundance but tended to decrease $(P<0.10)$ Firmicutes abundance in the ileum. Besides, $C$. butyricum addition resulted in higher $(P<0.05)$ abundances of Clostridia (Clostridiales) and Prevotellaceae, concurrent with an increasing trend $(P<0.10)$ of Bifidobacteriaceae abundance and decreased the abundances of several harmful bacteria such as Klebsiella $(P<0.05)$. Regarding ileal BA profile, there was a reduced $(P<0.05)$ content of tauro- $\alpha$-muricholic acid, increased $(P<0.05)$ contents of tauroursodeoxycholic acid and lithocholic acid, along with increasing trends $(P<0.10)$ of glycochenodeoxycholic acid and hyodeoxycholic acid contents due to $C$. butyricum addition, which also increased $(P<0.05)$ ileal FXR expression. Collectively, supplemental $C$. butyricum accelerated hepatic fatty acid oxidation, and shaped gut microbiota and BA profile, thus reducing fat deposition in the liver of aged laying hens.

Keywords: Clostridium butyricum, laying hen, lipid metabolism, gut microbiota, bile acid 


\section{INTRODUCTION}

Aged laying hens are known for their disturbance of lipid metabolism after having undergone the intensive metabolism at peak production (Boonsinchai, 2015; Liu et al., 2018), which could induce fat accumulation in the liver, thus leading to a defect of hepatic functionality of laying hens (Trott et al., 2014). Hence, there is a strong demand to regulate lipid metabolism of aged layers that could be critical for the practical production cost and animal welfare. Recent studies have focused on the importance of probiotics as a kind of regulator of lipid metabolism in chickens (Saleh et al., 2012, 2014). One of the potential mechanisms by which probiotics regulate lipid metabolism in chickens is through modifying the expression of lipogenesis-related genes such as acetyl-CoA carboxylase (ACC) and of lipolysis-related genes such as carnitine palmitoyltransferase in selected tissues such as muscle (Saleh et al., 2012, 2014). As an important constituent of probiotics, Clostridium butyricum principally colonizes in the distal small intestine and colon of animals (Sato and Tanaka, 1997). C. butyricum has been used to prevent or treat intestinal disorders of animals (Hayashi et al., 2013). Beyond the positive effects on intestinal tract, C. butyricum addition was reported recently to regulate lipid metabolism in various tissues of broiler chickens (Zhang et al., 2011; Zhao et al., 2013). However, no information is available regarding the effects of C. butyricum addition on lipid metabolism of laying hens.

Gut microbiota emerge as a crucial factor impacting the bioavailability of dietary components and exert a close connection with host nutritional and physiological processes, interacting with lipid metabolism and the development of obesity and fatty liver disorders of host (Lu et al., 2019; Monk et al., 2019; Zhao et al., 2019). There are obvious changes in gut microbial composition of laying hens with aging (Videnska et al., 2014). For aged hens, the gut microbiota dysbiosis was characterized by declined abundances of several beneficial bacteria (Videnska et al., 2014), which might be associated with the perturbation in their lipid metabolism (Jia et al., 2017). Previous studies have demonstrated that dietary C. butyricum could manipulate gut microbiota in chickens, as exhibited by the increased quantities of some beneficial bacteria and decreased quantities of certain harmful bacteria in broiler gut (Zhao et al., 2013; Zhang et al., 2014). Besides, the hepatoprotective roles of C. butyricum in mice have been suggested to be linked with its remodeling on gut microbial composition (Liu et al., 2017). Accordingly, C. butyricum addition might regulate lipid metabolism of chickens through shaping gut microbiota.

\footnotetext{
Abbreviation:ACC, acetyl-CoA carboxylase; BA, bile acid; FAS, fatty acid synthetase; FFA, free fatty acid; FXR, farnesoid X receptor; GCDCA, glycochenodeoxycholic acid; GLP, glucagon-like peptide; HDCA, hyodeoxycholic acid; HDL-C, high-density lipoprotein cholesterol; LCA, lithocholic acid; LCAD, long-chain acyl-CoA dehydrogenase; LDA, linear discriminant analysis; LDLC, low-density lipoprotein cholesterol; LXR, liver X receptor, FXR, acyl-CoA oxidase (ACOX); NMDS, non-metric multidimensional scaling; PCoA, principal coordinates analysis; PPAR, peroxisome proliferator activated receptor; SREBP, sterol-regulatory element-binding protein; $\mathrm{T}_{3}$, triiodothyronine; $\mathrm{T}_{4}$, thyroxine; T $\alpha$ MCA, tauro- $\alpha$-muricholic acid; TC, total cholesterol; TG, triglyceride; TUDCA, tauroursodeoxycholic acid.
}

Microbial influence over bile acid (BA) processing represents an important mechanism through which gut microbiota exert impacts on host metabolism (Tang et al., 2019). BAs could serve as a kind of signaling molecules interacting with FXR that is highly expressed in enterohepatic tissues, influencing hepatic BA homeostasis and lipid metabolism of host through the interplay between gut and liver (Rajani and Jia, 2018; Tang et al., 2019). Since aging-related gut microbiota dysbiosis could facilitate the development of obesity and fatty liver disease presumably through modifications of the chemical and signaling properties of BAs (Fu et al., 2012; Carbajo-Pescador et al., 2019; Tang et al., 2019), there might be a deterioration of intestinal BA profile accompanied by gut microbiota dysbiosis in aged laying hens, thereby favoring the accumulation of fat in their livers. Previous researches have revealed alterations of BA contents in serum, liver and feces, concurrent with a shift in gut microbiota of host due to dietary C. butyricum (Sato et al., 2012; Seo et al., 2013), suggesting that $C$. butyricum treatment may elicit an alteration of intestinal BA composition. Nevertheless, there is a lack of understanding about the regulation of intestinal BA profile in chickens, by which gut microbiota manipulation could contribute to the potential modulation of lipid metabolism in laying hens as a result of C. butyricum addition.

Comprehensively, the present study was aimed at exploring if supplemental C. butyricum could be an approach for modulating lipid metabolism in aged laying hens by targeting intestinal microbiota and BA profile that mediate the gut-liver crosstalk.

\section{MATERIALS AND METHODS}

\section{Animals and Experimental Design}

The experimental animal protocols for this study were approved by the Animal Care and Use Committee of the Feed Research Institute of the Chinese Academy of Agricultural Sciences (approval no. FRI-CAAS20190902). A total of 192 aged (60week-old) Hy-Line Brown laying hens were divided into two groups with eight replicate cages (12 birds per replicate). These birds are being used in a separate study and that the birds used in the present study are just sub-samples. The initial body weight (control: $1.93 \pm 0.15 \mathrm{~kg}$; treatment: $1.91 \pm 0.12 \mathrm{~kg}$ ) and egg production (control: $87.50 \pm 4.45 \%$; treatment: $89.58 \pm 3.86 \%$ ) of birds were similar across all the replicates. All birds were acclimated to the diet and environment for 1 week. Afterward, birds were fed a basal diet without or with addition of $2.7 \mathrm{~g} / \mathrm{kg}$ C. butyricum $\left(1 \times 10^{9} \mathrm{CFU} / \mathrm{g}\right)$ throughout the trial period. The additive dose of $C$. butyricum was based on our previous doseresponse study (in review). The actual count $\left(9.3 \times 10^{8} \mathrm{CFU} / \mathrm{g}\right)$ of $C$. butyricum in this supplement was determined using spread plate method. The C. butyricum strain (HJCB998) was obtained from Huijia Biological Technology, Ltd. (Hangzhou, China). This supplement was firstly premixed with corn powder and then mixed in the compound feed. All birds were raised in three-tier battery cages and exposed to $16 \mathrm{~h}$ of light/d. Room temperature was maintained between 14 and $20^{\circ} \mathrm{C}$. All birds were allowed free access to the water and mash feed. Composition of the basal 
diet based on the National Research Council (National Research Council [NRC], 1994) is shown in Table 1.

\section{Sample Collection}

At the end of week 8 of the experiment, one bird was randomly selected from each replicate cage $(n=8)$ and individual blood were taken aseptically from the wing vein after starvation for $10 \mathrm{~h}$. Serum samples were then obtained by centrifugation of blood at 3,000 rpm for $10 \mathrm{~min}$ at $4^{\circ} \mathrm{C}$ and stored at $-20^{\circ} \mathrm{C}$. These birds were slaughtered rapidly after blood collection, a little patch of the liver from each bird was immediately removed into RNAStore solution (TIANGEN Biotech., Co. Ltd., Beijing, China) and stored at $-80^{\circ} \mathrm{C}$ for RNA extraction. The remainder liver sample was collected for determination of hepatic lipids contents. Afterward, the intestinal tract of each bird was separated and the mid-point of ileal segment was then removed into RNAStore solution (TIANGEN Biotech., Co. Ltd., Beijing, China) and stored at $-80^{\circ} \mathrm{C}$ for RNA extraction. Besides, digesta sample in the ileum was collected and quick-froze by liquid nitrogen, followed by storage at $-80^{\circ} \mathrm{C}$.

\section{Biochemical Assay of Serum Samples}

Serum triglyceride (TG), total cholesterol (TC), high density lipoprotein cholesterol (HDL-C), and low density lipoprotein cholesterol (LDL-C) concentrations were measured using a KHB400 Automatic Biochemical Analyzer (Kehua Bioengineering, Co. Ltd., Shanghai, China). The ratio of HDL-C level to LDL-C level was calculated. Free fatty acids (FFAs) were quantified by colorimetry according to the protocols

TABLE 1 | Composition of the basal diet (air-dry basis).

\begin{tabular}{lc}
\hline Ingredients & Content (\%) \\
\hline Corn & 64.52 \\
Soybean meal (44\% crude protein) & 24.5 \\
Limestone & 8.9 \\
Sodium chloride & 0.3 \\
Dicalcium phosphate & 1.35 \\
Choline chloride (50\%) & 0.1 \\
DL-Methionine (98\%) & 0.1 \\
Multimineral & \\
Multivitamin & \\
Nutrient levels & 0.2 \\
Metabolizable energy (MJ/kg) & 0.03 \\
Crude protein (\%) & \\
Crude fat (\%) & 11.18 \\
Total phosphorus (\%) & 16.16 \\
Available phosphorus (\%) & 2.79 \\
Calcium (\%) & 0.54 \\
Lysine (\%) & 0.34 \\
Methionine (\%) & 3.5 \\
\hline
\end{tabular}

${ }^{1}$ Supplied per kilogram of diet: $\mathrm{Cu}, 8 \mathrm{mg}$; Zn, $66 \mathrm{mg}$; Fe, $60 \mathrm{mg}$; Mn, $65 \mathrm{mg}$; Se, $0.3 \mathrm{mg} ; \mathrm{l}, 1 \mathrm{mg}$. ${ }^{2}$ Supplied per kilogram of diet: vitamin A, 12,500 IU; vitamin $D_{3}$, 4,125 IU; vitamin E, $15 \mathrm{IU}$; menadione, $2 \mathrm{mg}$; thiamin, $1 \mathrm{mg}$; riboflavin, $8.5 \mathrm{mg}$; pyridoxine, $8 \mathrm{mg}$; cobalamin, $5 \mathrm{mg}$; pantothenic acid, $50 \mathrm{mg}$; niacin, $32.5 \mathrm{mg}$; biotin, $2 \mathrm{mg}$; folic acid, $5 \mathrm{mg}$; choline, $500 \mathrm{mg}$. of a commercial kit (Jiancheng Bioengineering Institute, Nanjing, China). The concentrations of lipid metabolism-related hormones including glucagon, insulin, glucagon-like peptide 1 (GLP-1), leptin, triiodothyronine $\left(\mathrm{T}_{3}\right)$ and thyroxine $\left(\mathrm{T}_{4}\right)$ in serum were determined by radioimmunoassay (Huaying Biotechnology, Co. Ltd., Beijing, China).

\section{Measurement of Hepatic Lipid Contents}

Total lipid was extracted from the liver sample that was preweighed, according to the method described by Folch et al. (1957). The dried liver lipid was then weighed to calculate the content of hepatic total lipid, which was expressed as the percentage of the weight of dried liver lipid to the wet weight of liver sample. The dried lipid was then dissolved in $2 \mathrm{~mL}$ butanol for measuring TC and TG contents with a VersaMax microplate reader (Molecular Devices, San Jose, CA, United States) by using commercial kits (Jiancheng Bioengineering Institute, Nanjing, China). In addition, another set of the liver samples were homogenized with cold saline. After centrifugation at $3000 \mathrm{rpm}$ for $10 \mathrm{~min}$ at $4^{\circ} \mathrm{C}$, the resultant supernatants were collected and FFAs contents were assayed colorimetrically using a commercial kit (Jiancheng Bioengineering Institute, Nanjing, China).

\section{RNA Isolation and Real-Time PCR}

Total RNA was extracted from the liver and ileum samples by using Trizol Reagent (TIANGEN Biotech., Co. Ltd., Beijing, China) following the manufacturer's instructions. The relative mRNA expression was quantified as previously described (Wang et al., 2019). Primer sequences for the reference gene (reduced glyceraldehyde-phosphate dehydrogenase) and target genes including ACC, fatty acid synthetase (FAS), sterolregulatory element-binding protein 1c (SREBP1c), liver $\mathrm{X}$ receptor $\alpha(\mathrm{LXR} \alpha)$, farnesoid $\mathrm{X}$ receptor (FXR), acyl-CoA oxidase (ACOX), long-chain acyl-CoA dehydrogenase (LCAD), peroxisome proliferator activated receptor $\alpha(\operatorname{PPAR} \alpha)$ and PPAR $\gamma$ are exhibited in Supplementary Table 1. The results of relative expression of genes were calculated using the $2^{-\Delta \Delta \mathrm{Ct}}$ method.

\section{High-Throughput Sequencing of Gut Microbiota}

Bacterial genomic DNA was extracted from ileal digesta using NucleoSpin ${ }^{\circledR}$ DNA Stool kit (MACHEREY-NAGEL Company, Germany). The quality of DNA samples were checked with gel electrophoresis. Bacterial 16S rDNA sequences spanning the variable regions $\mathrm{V} 3-\mathrm{V} 4$ were amplified according to the method described previously (Wang et al., 2019). The sequencing of the PCR products was performed on an Illumina HiSeq2500 PE250 platform (Illumina, San Diego, CA, United States). The sequencing results has been submitted to the Sequence Read Archive of the NCBI (Accession no. SAMN13610217). All the effective reads were clustered into operational taxonomic units (OTUs) based on a 97\% sequence similarity. Classification of OTUs at various taxonomic levels were implemented by comparing sequences to the GreenGene database. Microbial $\alpha$-diversity was analyzed using 
the MOTHUR v1.31.2 program (Schloss et al., 2009). The principal co-ordinates analysis ( $\mathrm{PCoA}$ ) and plot and non-metric multidimensional scaling (NMDS) were used to assess pairwise distances among samples ( $\beta$-diversity). Linear discriminant analysis (LDA) combined effect size measurements (LEfSe) was applied to identify the relative richness (LDA $>2, P<0.05)$ of bacteria between groups.

\section{Targeted Metabolome Analysis of Intestinal BAs}

The ultra high performance liquid chromatography-mass spectrometry (UPLC/MS) method was modified to determine BAs contents in ileal digesta (Tang et al., 2019). Briefly, approximately $50 \mathrm{mg}$ of each sample was deproteinized by adding $1000 \mu \mathrm{L}$ of extract solvent (Vacetonitrile: Vmethanol: Vwater $=2: 2: 1$, containing $0.1 \%$ formic acid and $50 \mathrm{nmol} / \mathrm{L}$ internal standard). The mixture was vortexed for $30 \mathrm{~s}$, homogenized at $35 \mathrm{~Hz}$ for $4 \mathrm{~min}$, and sonication for $5 \mathrm{~min}$ in ice-water bath. These steps were repeated for three times, followed by incubation at $-20^{\circ} \mathrm{C}$ for $1 \mathrm{~h}$. After centrifugation at $11,000 \mathrm{rpm}$ for $15 \mathrm{~min}$ at $4^{\circ} \mathrm{C}$, the resulting supernatant was applied to the UPLC/MS system. The BAs were quantified by a 1290 Infinity series UHPLC System (Agilent Technologies, United States) coupled with a Q Exactive ${ }^{\mathrm{TM}}$ Focus MS (Thermo Fisher Scientific, United States). An ACQUITY UPLC BEH column $(150 \mathrm{~mm} \times 2.1 \mathrm{~mm}, 1.7 \mu \mathrm{m}$, Waters $)$ heated to $45^{\circ} \mathrm{C}$ was used for chromatographic separation. A gradient system consisted of the Solvent A $(0.1 \%$ acetic acid) and the Solvent $B$ (acetonitrile) at a flow rate of $0.3 \mathrm{~mL} / \mathrm{min}$. The auto-sampler temperature was set at $4^{\circ} \mathrm{C}$ and the injection volume was $3 \mu \mathrm{L}$. The ion source parameter was set as follows: spray voltage $=+3500 /-3100 \mathrm{~V}$, sheath gas flow rate $=40 \mathrm{~L} / \mathrm{h}$, aux gas flow rate $=15 \mathrm{~L} / \mathrm{h}$, aux gas temperature $=350^{\circ} \mathrm{C}$, and capillary temperature $=320^{\circ} \mathrm{C}$. BA standards were obtained from Sigma-Aldrich, Co. Ltd. (St. Louis, MO, United States). The mix reference standards were prepared by dissolving each BA reference standard respectively in methanol.

\section{Statistical Analysis}

Data are presented as mean \pm standard deviation and analyzed by using SPSS 18.0 software. $t$-Tests were used to detect effects of dietary treatment on serum and hepatic biochemical indices, hepatic and intestinal genes expression, as well as intestinal BAs contents. Wilcoxon rank tests were used to detect the differences in the relative abundances of bacteria between groups. Significance was defined as $P<0.05$ and $0.05 \leq P<0.10$ was considered to be a tendency toward significance.

\section{RESULTS}

\section{Lipid and Hormone Concentrations in Serum}

Clostridium butyricum addition did not significantly alter $(P>0.10)$ serum TC, HDL-C, LDL-C, and FFAs concentrations of laying hens (Table 2), but tended to reduce $(P=0.091)$ serum TG concentration. Birds consumed a diet containing C. butyricum had significant higher $(P<0.05)$ concentrations of GLP-1, insulin, $\mathrm{T}_{3}$ and $\mathrm{T}_{4}$ in serum as compared with birds received a control diet (Table 3 ).

\section{Hepatic Lipid Contents, and Gene Expression in the Liver and Intestine}

Clostridium butyricum treatment had no significant impacts $(P>0.10)$ on the contents of total lipid, TC and FFAs in the liver of birds (Table 4), but led to a decreasing trend $(P=0.062)$ of TG content with significantly reduced $(P<0.05)$ contents of FFAs in the liver. In terms of lipid metabolism-related genes, significantly increased $(P<0.05)$ mRNA expression of FAS, FXR, ACOX, and $\operatorname{PPAR} \alpha$ were observed in the liver of birds supplemented with C. butyricum (Figure 1), which also significantly up-regulated $(P<0.05)$ FXR expression in the ileum.

\section{Diversity of Gut Microbiota}

No significant changes $(P>0.10)$ occurred in the $\alpha$-diversity including abundance-based coverage estimator (ACE) and Chao 1

TABLE 2 | Effects of Clostridium butyricum treatment on serum lipid concentrations ${ }^{1}$ of laying hens.

\begin{tabular}{|c|c|c|c|c|c|c|}
\hline & TC (mmol/L) & $\mathrm{TG}(\mathrm{mmol} / \mathrm{L})$ & HDL-C (mmol/L) & LDL-C (mmol/L) & $H / L$ & FFAs (mmol/L) \\
\hline Control & $3.09 \pm 0.64$ & $4.04 \pm 0.68$ & $1.14 \pm 0.42$ & $0.38 \pm 0.15$ & $3.23 \pm 1.02$ & $0.39 \pm 0.11$ \\
\hline Treatment & $3.46 \pm 0.53$ & $3.47 \pm 0.57$ & $1.12 \pm 0.29$ & $0.31 \pm 0.10$ & $3.69 \pm 0.63$ & $0.33 \pm 0.12$ \\
\hline$P$-value & 0.230 & 0.091 & 0.913 & 0.335 & 0.296 & 0.331 \\
\hline
\end{tabular}

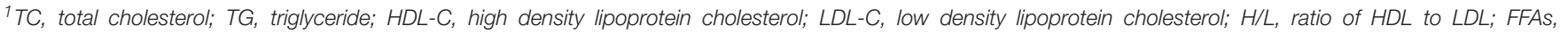
free fatty acids.

TABLE 3 | Effects of Clostridium butyricum treatment on serum hormones ${ }^{1}$ concentrations of laying hens.

\begin{tabular}{|c|c|c|c|c|c|c|}
\hline & GLP-1 (pmol/L) & Glucagon (pg/mL) & Insulin ( $\mu$ IU/mL) & Leptin (ng/mL) & $\mathrm{T}_{3}(\mathrm{ng} / \mathrm{mL})$ & $\mathrm{T}_{4}(\mathrm{ng} / \mathrm{mL})$ \\
\hline Control & $21.06 \pm 3.69^{b}$ & $61.77 \pm 7.62$ & $10.10 \pm 1.45^{b}$ & $4.15 \pm 0.63$ & $0.94 \pm 0.07^{b}$ & $15.41 \pm 1.44^{b}$ \\
\hline Treatment & $26.62 \pm 5.99^{a}$ & $67.84 \pm 7.68$ & $12.38 \pm 1.74^{\mathrm{a}}$ & $4.57 \pm 0.58$ & $1.19 \pm 0.15^{\mathrm{a}}$ & $19.49 \pm 1.71^{\mathrm{a}}$ \\
\hline$P$-value & 0.042 & 0.135 & 0.031 & 0.196 & 0.001 & $<0.001$ \\
\hline
\end{tabular}

${ }^{a, b}$ Values with different superscripts within the same column differ significantly $(P<0.05) .{ }^{1} G L P-1$, glucagon-like peptide 1 ; $T_{3}$, triiodothyronine; $T_{4}$, tetraiodothyronine. 
TABLE 4 | Effects of Clostridium butyricum treatment on hepatic lipid contents ${ }^{1}$ of laying hens.

\begin{tabular}{lcccc}
\hline & $\begin{array}{c}\text { Total lipid } \\
\text { content }(\%)\end{array}$ & $\begin{array}{c}\text { TC }(\boldsymbol{\mu} \text { mol} / \mathbf{g} \\
\text { tissue) }\end{array}$ & $\begin{array}{c}\text { TG ( } \boldsymbol{\mu ~ m o l} / \mathbf{g} \\
\text { tissue) }\end{array}$ & $\begin{array}{c}\text { FFAs }(\boldsymbol{\mu ~ m o l} / \mathbf{g} \\
\text { tissue) }\end{array}$ \\
\hline Control & $13.57 \pm 2.54$ & $37.61 \pm 5.58$ & $90.75 \pm 16.09$ & $6.66 \pm 1.17^{\mathrm{a}}$ \\
Treatment & $16.08 \pm 5.70$ & $38.31 \pm 6.76$ & $74.34 \pm 10.37$ & $5.16 \pm 1.35^{\mathrm{b}}$ \\
P-value & 0.348 & 0.849 & 0.062 & 0.032
\end{tabular}

a,b Values with different superscripts within the same column differ significantly $(P<0.05)$. ${ }^{1}$ Total lipid content was expressed as the percentage of the weight of dried liver lipid to the wet weight of liver sample. TC, total cholesterol; TG, triglycerides; FFAs, free fatty acids.

estimator along with Shannon and Simpson indexes of ileal microbiota of birds in response to $C$. butyricum addition (Supplementary Table 2). There were significant separations $(P<0.05)$ of ileal microbial communities between groups, as visualized by both PCoA and NMDS plots (Figure 2).

\section{Composition of Gut Microbiota}

The dominated phylum in the ileum of birds was Firmicutes, which contributed greater than $95 \%$ of the whole phyla (Supplementary Figure 1A). Other phyla were presented at very low relative abundances. The dominated class in the microbiota was Bacilli, followed by Actinobacteria (Supplementary Figure 1B). Within Bacilli, the majority belonged to the order Lactobacillales (mainly comprised the family Lactobacteriaceae), while the main order within Actinobacteria was Bifidobacteriales (Supplementary Figure 1C). Family level analysis showed that the microbiota was dominated by Lactobacillaceae, followed by Bifidobacteriaceae (Supplementary Figure 1D). At genus level, the Lactobacillus followed by Aeriscardovia accounted for the largest proportion of the microbiota (Supplementary Figure 1E).

The Wilcoxon rank tests identified a number of bacteria at various taxonomic levels as biomarkers to distinguish groups (Table 5). Treating birds with C. butyricum significantly elevated $(P<0.05)$ the abundances of Bacteroidetes and Clostridia (Clostridiales), and tended to increase $(P=0.061)$ Bacteroidia abundance, as well as tended to reduce $(P<0.10)$ the abundances of Firmicutes and Bacilli. C. butyricum treatment also significantly lowered $(P<0.05)$ the abundances of Bacillaceae (Bacillus) and tended to reduce $(P=0.088)$ Lactobacillales abundance, but showed a tendency to increase $(P=0.078)$ the abundances of Bifidobacteriales (Bifidobacteriaceae). There was a decreasing trend $(P=0.068)$ of Enterobacteriaceae abundance with a significant reduction $(P<0.05)$ of Klebsiella abundance in treatment group compared with control. Moreover, C. butyricum treatment induced significant elevations $(P<0.05)$ in the abundances of Corynebacteriaceae (Corynebacterium), Psychrobacter and Rothia, along with increasing trends $(P<0.10)$ of the abundances of Brevibacteriaceae (Brevibacterium). LEfSe analysis revealed enrichments (LDA $>2, P<0.05$ ) of several other beneficial bacteria such as the family Prevotellaceae and the genus Alloprevotella in the ileum of treatment group (Figure 3). Remarkably, the microbiota from treatment group was also enriched (LDA $>2, P<0.05$ ) with the genus Clostridium sensu
TABLE 5 | Differences of bacterial distribution (\%) in ileal digesta between the control and Clostridium butyricum treatment groups.

\begin{tabular}{|c|c|c|c|}
\hline & Control & Treatment & $P$-value \\
\hline \multicolumn{4}{|l|}{ Phyla } \\
\hline Bacteroidetes & $0.13 \pm 0.04^{b}$ & $0.20 \pm 0.09^{a}$ & 0.044 \\
\hline Firmicutes & $98.70 \pm 0.84$ & $95.06 \pm 4.87$ & 0.061 \\
\hline \multicolumn{4}{|l|}{ Classes } \\
\hline Clostridia & $0.35 \pm 0.13^{b}$ & $0.92 \pm 0.52^{a}$ & 0.011 \\
\hline Bacilli & $98.30 \pm 0.90$ & $94.10 \pm 5.14$ & 0.055 \\
\hline Bacteroidia & $0.13 \pm 0.04$ & $0.20 \pm 0.09$ & 0.061 \\
\hline \multicolumn{4}{|l|}{ Orders } \\
\hline Cardiobacteriales & $0.002 \pm 0.002^{b}$ & $0.051 \pm 0.010^{a}$ & $<0.001$ \\
\hline Bacillales & $0.395 \pm 0.160^{a}$ & $0.011 \pm 0.006^{b}$ & $<0.001$ \\
\hline Micrococcales & $0.021 \pm 0.009^{b}$ & $0.066 \pm 0.031^{a}$ & 0.004 \\
\hline Clostridiales & $0.349 \pm 0.126^{b}$ & $0.916 \pm 0.516^{a}$ & 0.018 \\
\hline Enterobacteriales & $0.082 \pm 0.042$ & $0.048 \pm 0.019$ & 0.059 \\
\hline Bifidobacteriales & $0.510 \pm 0.646$ & $2.474 \pm 1.162$ & 0.078 \\
\hline Lactobacillales & $97.94 \pm 0.99$ & $94.12 \pm 5.14$ & 0.088 \\
\hline \multicolumn{4}{|l|}{ Families } \\
\hline Wohlfahrtiimonadaceae & $0.002 \pm 0.002^{b}$ & $0.051 \pm 0.010^{a}$ & $<0.001$ \\
\hline Bacillaceae & $0.395 \pm 0.160^{a}$ & $0.010 \pm 0.007^{b}$ & $<0.001$ \\
\hline Corynebacteriaceae & $0.014 \pm 0.007^{b}$ & $0.027 \pm 0.007^{a}$ & 0.012 \\
\hline Micrococcaceae & $0.009 \pm 0.003^{b}$ & $0.049 \pm 0.031^{a}$ & 0.012 \\
\hline Carnobacteriaceae & $0.017 \pm 0.009^{b}$ & $0.034 \pm 0.014^{a}$ & 0.030 \\
\hline Clostridiaceae & $0.166 \pm 0.072$ & $0.629 \pm 0.548$ & 0.060 \\
\hline Enterobacteriaceae & $0.082 \pm 0.042$ & $0.048 \pm 0.019$ & 0.068 \\
\hline Brevibacteriaceae & $0.001 \pm 0.002$ & $0.002 \pm 0.002$ & 0.072 \\
\hline Bifidobacteriaceae & $0.510 \pm 0.646$ & $2.474 \pm 1.162$ & 0.078 \\
\hline \multicolumn{4}{|l|}{ Genera } \\
\hline Ignatzschineria & $0.002 \pm 0.002^{b}$ & $0.051 \pm 0.010^{a}$ & $<0.001$ \\
\hline Bacillus & $0.395 \pm 0.160^{a}$ & $0.010 \pm 0.007^{b}$ & $<0.001$ \\
\hline Brachybacterium & $0.001 \pm 0.002^{b}$ & $0.010 \pm 0.003^{a}$ & $<0.001$ \\
\hline Psychrobacter & $0.001 \pm 0.001^{b}$ & $0.008 \pm 0.003^{a}$ & 0.001 \\
\hline Corynebacterium & $0.008 \pm 0.006^{b}$ & $0.017 \pm 0.005^{a}$ & 0.011 \\
\hline Rothia & $0.008 \pm 0.002^{b}$ & $0.043 \pm 0.028^{a}$ & 0.013 \\
\hline Klebsiella & $0.036 \pm 0.026^{a}$ & $0.006 \pm 0.004^{b}$ & 0.013 \\
\hline Terrisporobacter & $0.055 \pm 0.022^{a}$ & $0.031 \pm 0.010^{b}$ & 0.025 \\
\hline Clostridium sensu stricto 13 & $0.008 \pm 0.006$ & $0.522 \pm 0.575$ & 0.054 \\
\hline Brevibacterium & $0.001 \pm 0.002$ & $0.002 \pm 0.002$ & 0.079 \\
\hline
\end{tabular}

stricto 13 , which is probably derived from the increase of species C. butyricum in the ileum.

\section{Intestinal BA Profile}

The ion recording chromatographs showed excellent peak shapes with good separations among BA standard solutions (Supplementary Figure 2). Orthogonal partial least squares discrimination analysis (OPLS-DA) revealed an obvious separation of ileal BA profile between groups (Figure 4). Chenodeoxycholic acid, taurochenodeoxycholic acid, and cholic acid accounted for the largest proportion of primary BAs in the ileum of birds (Table 6), while allocholic acid, $\lambda$-muricholic acid, and 7-ketolithocholic acid represented the major secondary BAs in the ileum. There was a significant reduced $(P<0.05)$ content of tauro- $\alpha$-muricholic acid (T $\alpha$ MCA) with a decreasing 
trend $(P=0.065)$ of taurohyodeoxycholic acid content in the ileum due to $C$. butyricum treatment, which also significantly elevated $(P<0.05)$ the contents of tauroursodeoxycholic acid (TUDCA), lithocholic acid (LCA) and 7-ketolithocholic acid, as well as tended to increase $(P<0.10)$ the contents of glycochenodeoxycholic acid (GCDCA) and hyodeoxycholic acid (HDCA) in the ileum.

\section{DISCUSSION}

Serum lipid and lipoprotein concentrations are indicative of the basal adjustment of fatty acid (FA) circulation between the liver and peripheral tissues (Mossab et al., 2002). LDL is the main carrier of circulating FAs and cholesterol from the liver to extrahepatic tissues, and LDL-C serves as a risk factor for atherosclerosis (Jacobson et al., 2012). Contrastively, HDL carries FAs and cholesterol from peripheral tissues to the liver for catabolism, and HDL-C functions as an indicator of excretion of the surplus cholesterol to gut (Jacobson et al., 2012). Accumulating studies have indicated that C. butyricum addition tended to decrease serum TG and TC contents, but failed to modify serum lipoprotein cholesterol level in broilers (Zhang et al., 2011; Zhao et al., 2013). Similarly, the present study showed that administration of $C$. butyricum into layer diet resulted in a decreasing trend of TG level without an overall alteration of lipid profile in serum. It is well-known that lipid metabolism is strongly regulated by a variety of hormones, in which glucagon and insulin have negative and positive regulations on lipogenesis, respectively (Li et al., 2019). Leptin that produced by adipose tissue is related to the negative regulation of lipid deposition (Li et al., 2019). Thyroid hormones such as $\mathrm{T}_{3}$ and $\mathrm{T}_{4}$ are the main hormones involved in the catabolic processes of lipids (Li et al., 2019). GLP-1 is an intestinal signal peptide hormone that capable of regulating glucose-dependent secretion of insulin, but also elicits a promotion of lipid oxidation (Mulvihill, 2018). It was reported that $C$. butyricum addition increased serum insulin level of broilers (Zhao et al., 2013), and promoted GLP1 secretion in mice (Jia et al., 2017). Herein, C. butyricum addition elevated serum insulin level, together with GLP-1, $\mathrm{T}_{3}$ and $\mathrm{T}_{4}$ levels, implying a promotion of lipogenesis with a simultaneous enhancement of lipolysis in laying hens due to C. butyricum addition. Alternatively, the increased levels of GLP$1, \mathrm{~T}_{3}$ and $\mathrm{T}_{4}$ following C. butyricum addition might represent an adaptive response of the corresponding elevation of insulin (Li et al., 2019).

Probiotic strains have been suggested to elicit lipid-lowering effect via several underlying mechanisms such as modulating lipid metabolism-related gene expression and enzyme activities (Yoo et al., 2013). In the present study, the reduction of hepatic FFA content with the decreasing trend of hepatic TG content suggested a reduced lipid deposition in the liver of layers due to C. butyricum addition, which might cause a decreased risk of fatty liver syndrome (Trott et al., 2014). Lipid deposition inside the body is largely dependent on the balance between lipolysis and lipogenesis. Among genes involved in lipogenic processes, ACC is the first rate-limiting enzyme of de novo synthesis of FAs, FAS also functions as a key determinant

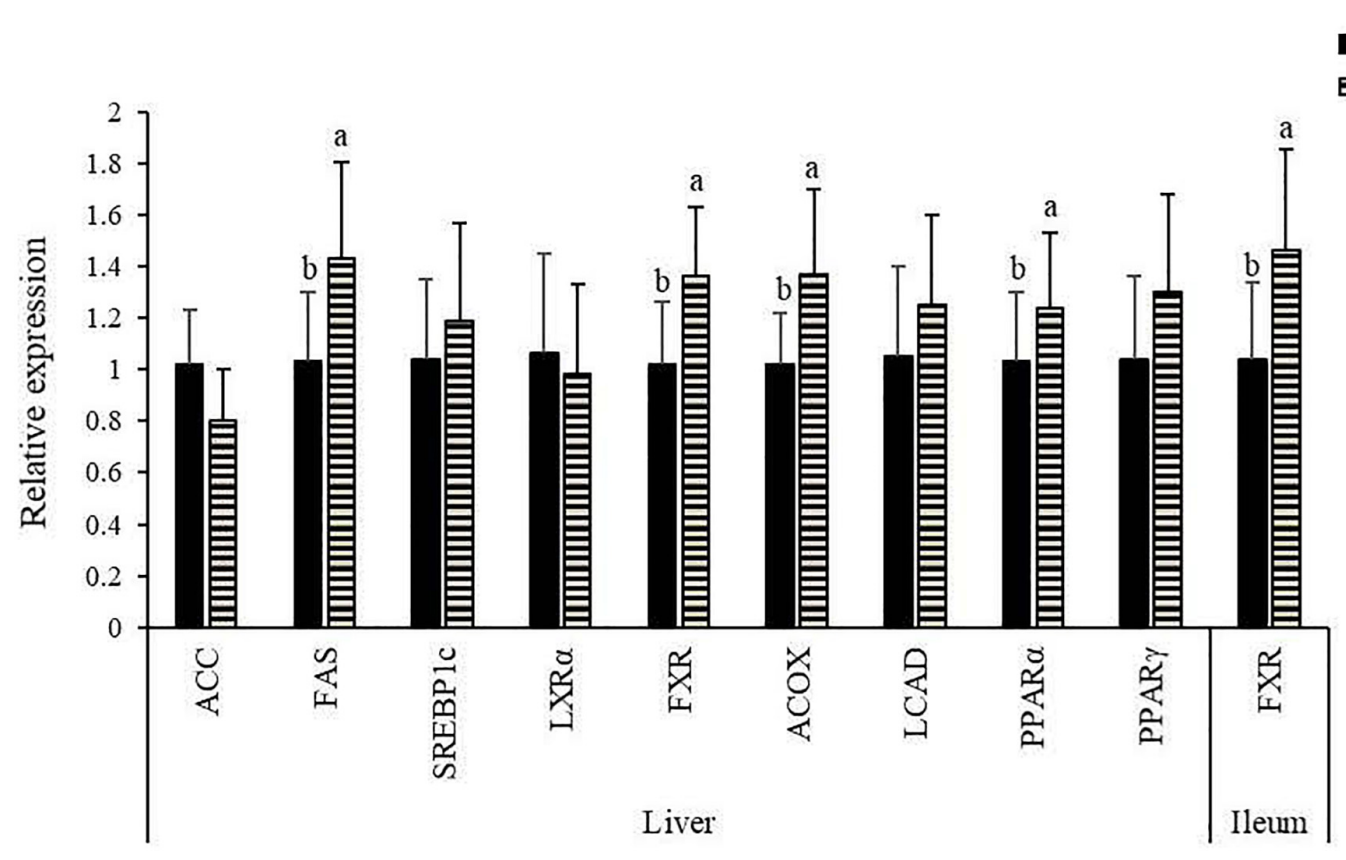

FIGURE 1 | Effects of Clostridium butyricum treatment on the relative mRNA expression of lipid metabolism-related genes ${ }^{1}$ in the liver and ileum of laying hens. ${ }^{a, b}$ Treatments with unlike letters were significantly different $(P<0.05) .{ }^{1} \mathrm{ACC}$, acetyl-CoA carboxylase; FAS, fatty acid synthetase; SREBP, sterol-regulatory element-binding protein; LXR, liver X receptor; FXR, farnesoid X receptor; ACOX, acyl-CoA oxidase; LCAD, long-chain acyl-CoA dehydrogenase; PPAR, peroxisome proliferator activated receptor. 
A

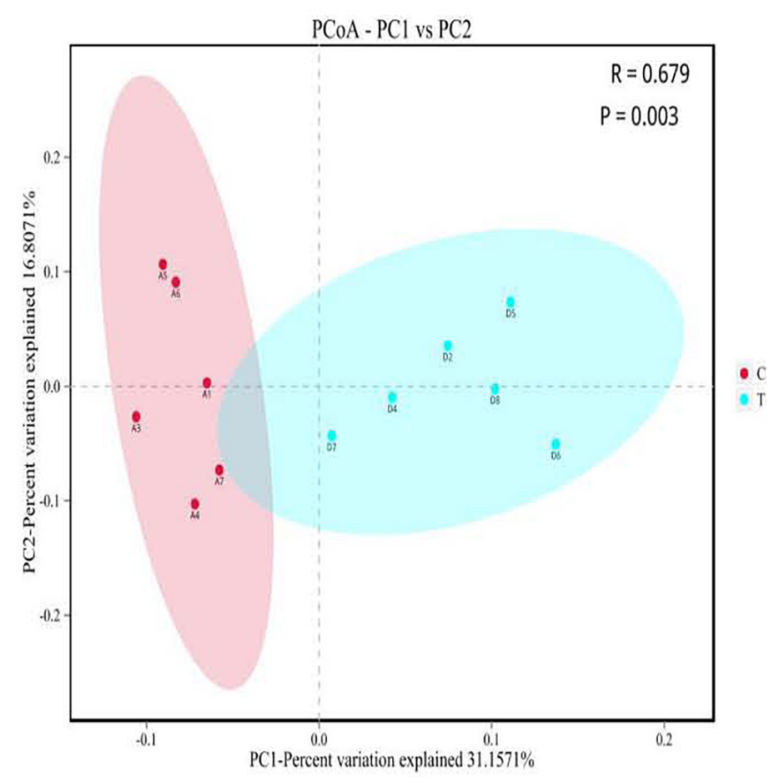

B

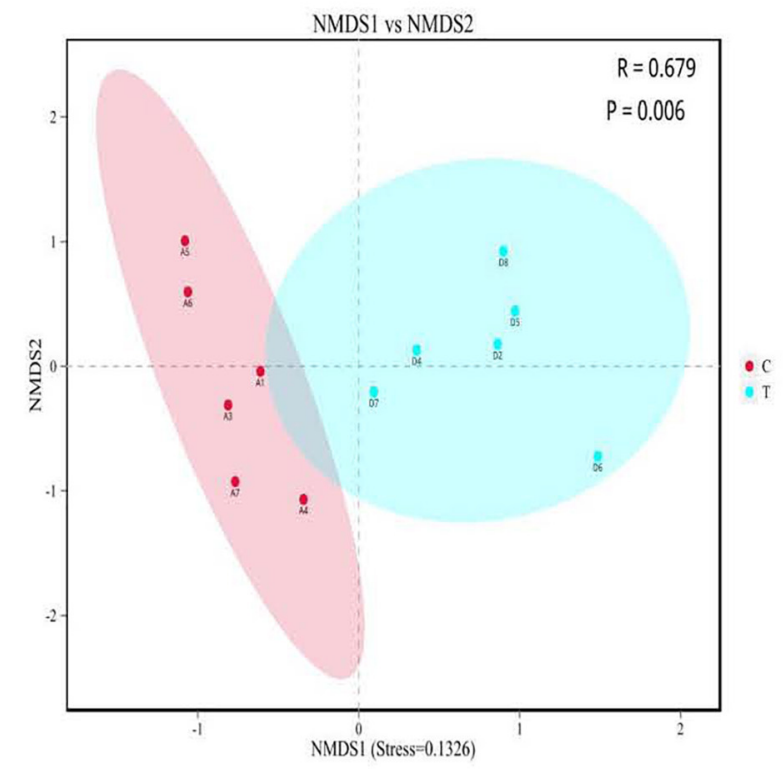

FIGURE 2 | Beta diversity analysis of gut microbiota between the control and Clostridium butyricum treatment groups. (A) Principal co-ordinates analysis (PCoA) plot; (B) Non-metric multidimensional scaling (NMDS) plot. C, control group; T, C. butyricum treatment group.

for the maximal capacity of lipogenesis, due to its role in catalyzing the elongation of carbon chains of FAs (Lu et al., 2018). In this study, hepatic FAS expression was increased in treatment group, which might result in a greater capacity of lipogenesis. This was in accordance with a previous study in broilers (Zhao et al., 2013). Since insulin secretion can activate the transcription of FAS by binding to its promoter (Griffin and Sul, 2004), the increased expression of FAS following C. butyricum addition was likely related to the elevated level of insulin. As a nuclear transcription factor binding to the sterol-regulatory element, SREBP1c facilitates biosynthesis of FAs and their incorporation into TG by directly promoting the expression of its target genes such as ACC and FAS (Horton et al., 2002). LXR $\alpha$ is a ligand-activated nuclear receptor with important functions in transcriptional control of FA synthesis by promoting SREBP1c expression and activating the promoters of ACC and FAS (Wang and Tontonoz, 2018). PPARs are molecular sensors of FAs and their derivatives. Among them, PPAR $\gamma$ is involved in lipogenic process by promoting the transcription of its target gene SREBP1c (Mirza and Sharma, 2019). In this study, no differences were detected in hepatic PPAR $\gamma$, $\mathrm{LXR} \alpha$ and SREBP1c expression between groups, suggesting that C. butyricum-induced up-regulation of FAS expression was not through the pathway of PPAR $\gamma-\mathrm{LXR} \alpha-\mathrm{SREBP} 1 \mathrm{c}$. As the ratelimiting enzymes in lipolytic processes, LCAD and ACOX are respectively associated with catalyzation of the initial steps of mitochondrial and peroxisomal FA $\beta$-oxidation (Chu et al., 1994; Knottnerus et al., 2018). ACOX is characterized by a PPAR response element and responds to a change in FA level in a PPAR $\alpha$-dependent manner (Ringseis and Eder, 2009; Knottnerus et al., 2018). FXR acts as a receptor of BAs, playing critical roles in regulating BA homeostasis and lipid metabolism (Fang et al., 2015; Rajani and Jia, 2018). A targeted disruption of FXR could induce lipid accumulation in both serum and liver (Sinal et al., 2000). In contrast, FXR activation could reduce hepatic lipids levels through the positive regulation of PPAR $\alpha$, highlighting a role of FXR in accelerating lipolysis (Rajani and Jia, 2018). Thus, the increased expression of hepatic ACOX, PPAR $\alpha$ and FXR in treatment group suggested that C. butyricum addition stimulated the peroxisomal FA $\beta$-oxidation probably through FXR-PPAR $\alpha$-ACOX pathway, thereby accounting for the corresponding reduced contents of hepatic FFAs and TG. Similarly, Weng et al. (2015) reported that C. butyricum addition promoted the peroxisomal $\beta$-oxidation of hepatic FAs via PPAR $\alpha$-ACOX pathway in mice. Seo et al. (2013) also observed an up-regulation of hepatic PPAR $\alpha$ expression due to C. butyricum addition, possibly benefiting the inhibition of lipid accumulation in rats.

Gut microbiota exert essential roles in contributing to the modulation of host lipid metabolism by dietary intervention (Lu et al., 2019; Ushiroda et al., 2019). It was found that obese animals consistently showed a decrease in Bacterioidetes and an increase in Firmicutes in gut (Lu et al., 2019). More studies have validated that the increase of Firmicutes with the reduction of Bacteroidetes in gut exert a strong linkage with host lipid accumulation and fatty liver (Fontaine et al., 2019; Ushiroda et al., 2019). Consequently, the increased abundance of Bacteroidetes with the decreasing trend of Firmicutes abundance could be at least partially responsible for the reduced lipid deposition in the liver of layers as a result of $C$. butyricum addition. The increase of Bacilli and the reduction of Bacteroidia in gut were implicated in 
A

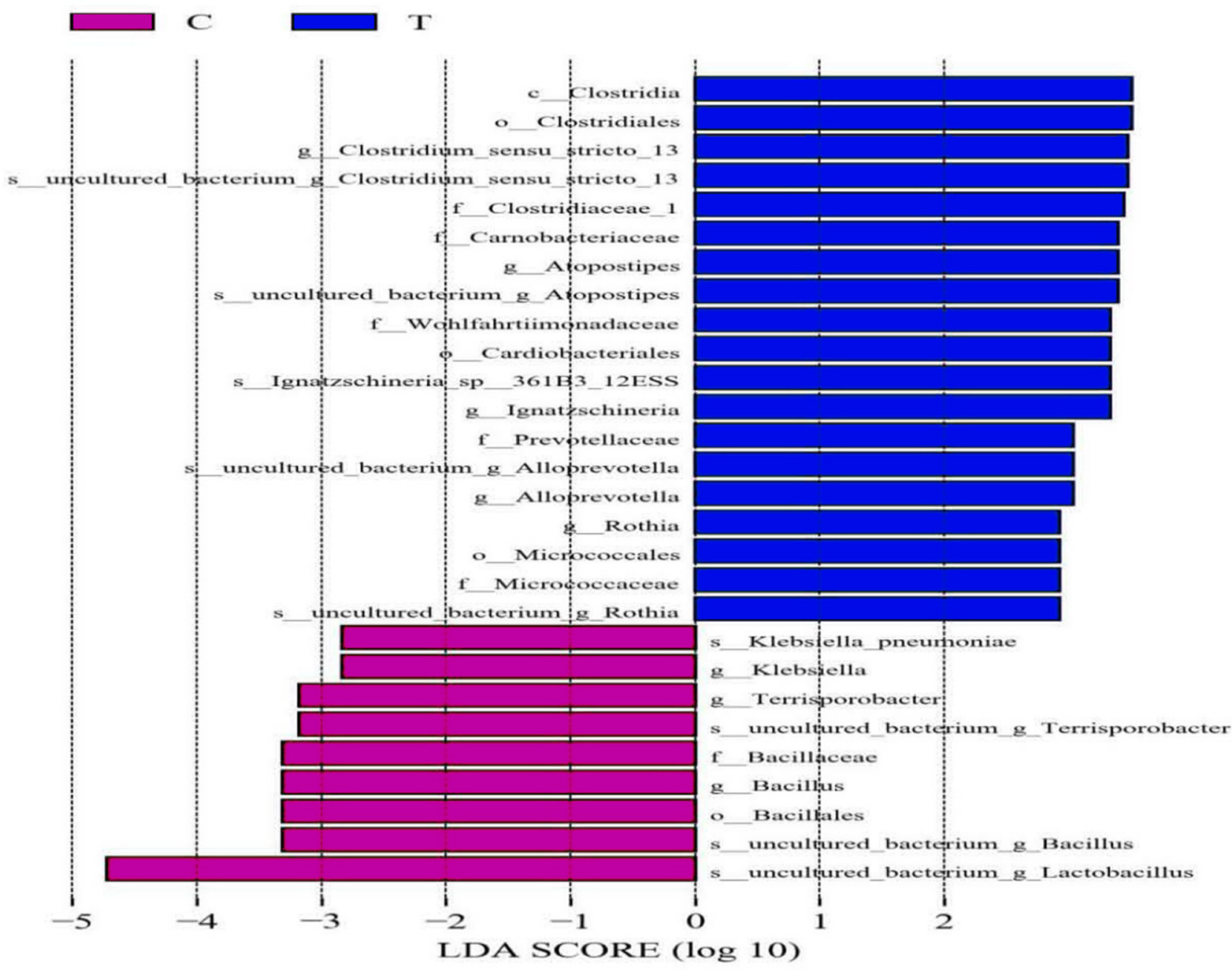

B
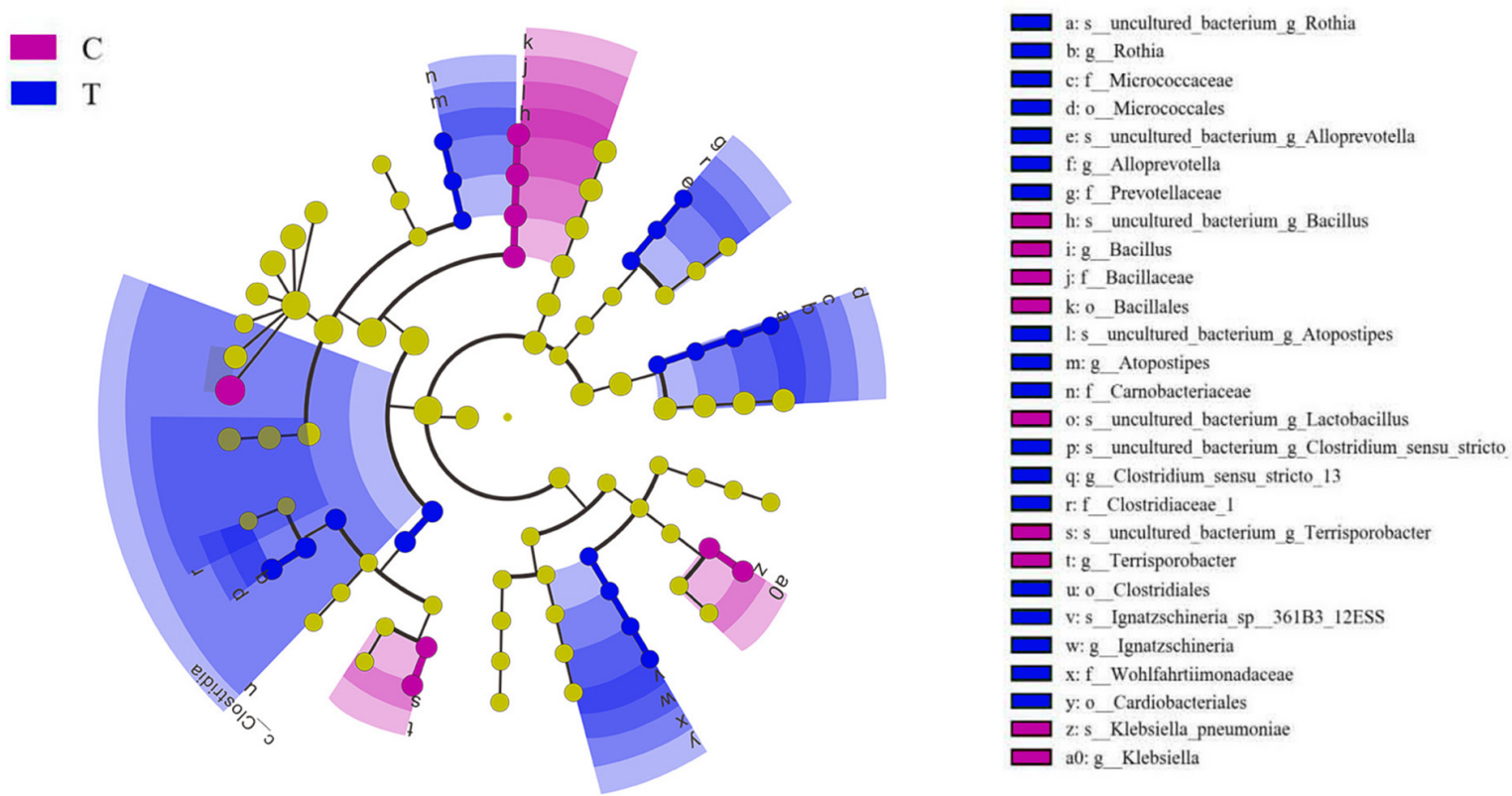

FIGURE 3 | Linear discriminant analysis (LDA) combined effect size measurements (LEfSe) analysis of ileal microbiota of laying hens. (A) Species with significant differences $(P<0.05)$ that have an LDA score greater than the estimated value (2.0). (B) The cladogram diagram shows the microbial species with significant differences between groups. The species classification at the levels of phylum, class, order, family, and genus exhibited from the inside to the outside. C, control group; T, Clostridium butyricum treatment group. 


\section{Scores (OPLS-DA)}

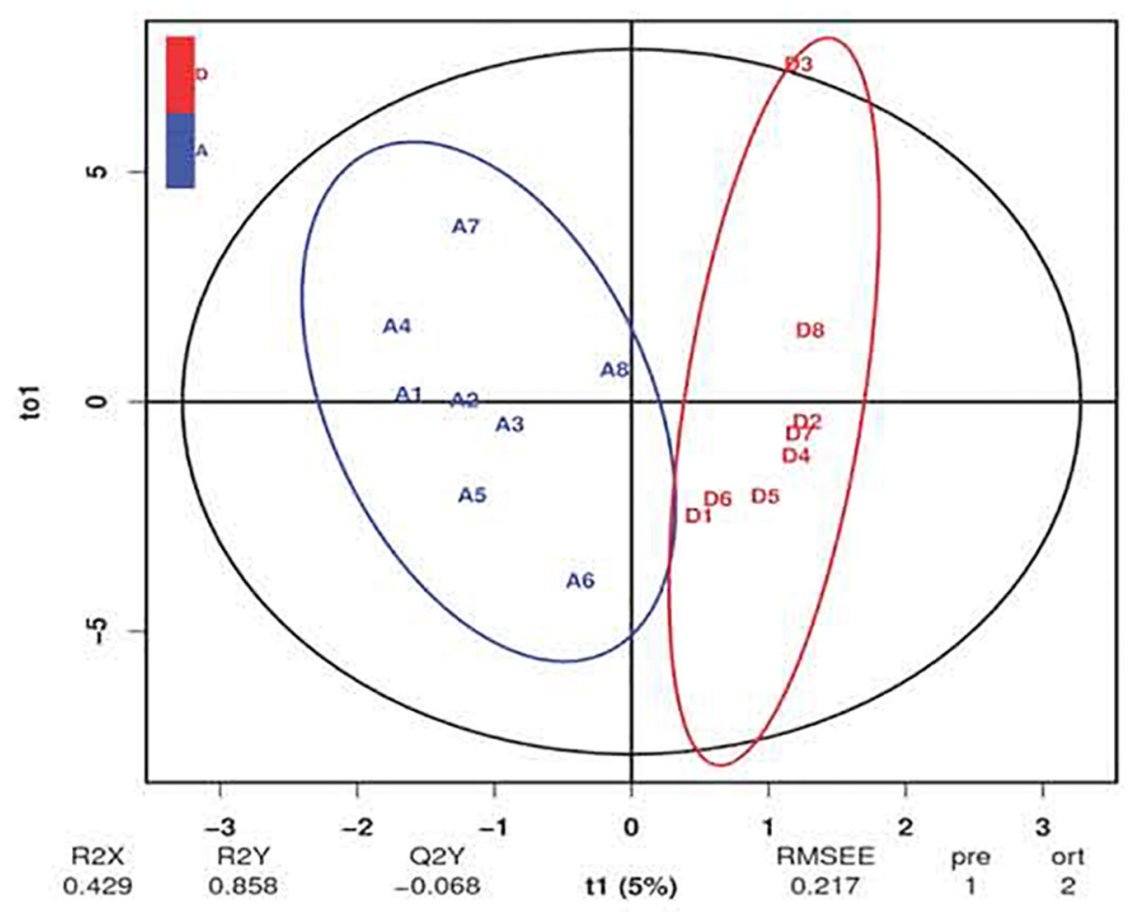

FIGURE 4 | Orthogonal partial least squares discrimination analysis (OPLS-DA) of ileal bile acid profile of laying hens. The blue letters represent the samples of control group, while the red letters represent the samples from Clostridium butyricum treatment group.

high-fat diet-induced obesity of animals (Fontaine et al., 2019; Ushiroda et al., 2019). Clostridia represents a critical class associated with short-chain fatty acids production that favors the inhibition of lipid accumulation and obesity (Murugesan et al., 2018). Within Clostridia, the order Clostridiales and the family Clostridiaceae were evidenced to be decreased in gut due to obesity (Monk et al., 2019; Zhao et al., 2019). In contrast, an increased abundance of Lactobacillales in gut was associated with obesity of animals (Fontaine et al., 2019; Lu et al., 2019). In this study, C. butyricum addition increased the abundances of Clostridia (including Clostridiales and Clostridiaceae) and Bacteroidia, as well as decreased Lactobacillales abundance and tended to reduce Bacilli abundance, which could be beneficial for decreasing lipid deposition in the liver of layers. Enterobacteriaceae especially the genus Klebsiella were identified as harmful bacteria with massive production of lipopolysaccharide (Keskitalo et al., 2018; Pope et al., 2019), which could trigger systemic inflammation and metabolic endotoxemia, contributing to the defective hepatic functions and lipid accumulation (Cani et al., 2007). As a well-known probiotic, Bifidobacterium was found to be reduced in the gut of obese animals (Wang et al., 2015). A previous study has confirmed the capability of Bifidobacterium treatment to suppress lipid accumulation and reduce the risk of obesity probably through attenuating endotoxemia (Wang et al., 2015). Herein, C. butyricum addition tended to elevate Bifidobacteriales (Bifidobacteriaceae) abundance but reduced Enterobacteriales and Klebsiella abundances, which might lower the susceptibility of lipid deposition and thus favor the reduction of lipid deposition in the liver. Similarly, Shang et al. (2016) reported that feeding $C$. butyricum attenuated endotoxemia of mice, through which $C$. butyricum inhibit lipid accumulation in the liver. As a producer of certain functional substances such as succinate, Prevotellaceae exerts a negative relationship with lipid deposition in animals (Zhang et al., 2019). A further study revealed reduced abundances of Prevotellaceae (Prevotella) and Alloprevotella in gut of animals with hepatic steatosis (Tang et al., 2019). Thereby, the enrichment of Prevotellaceae and Alloprevotella in C. butyricum treatment group might also favor the reduction of lipid deposition in the liver of laying hens.

BAs are well-known to possess metabolic functions in cholesterol homeostasis and lipid metabolism. The shift of BA profile induced by gut microbiota is closely associated with hepatic lipid accumulation of host (Tang et al., 2019). C. butyricum addition was reported to modify BAs contents in serum, liver and feces, as well as manipulate gut microbiota (Sato et al., 2012; Seo et al., 2013), suggesting that C. butyricum addition may shape intestinal BA profile of host. Indeed, the current study revealed that layers fed with $C$. butyricum had an alteration of ileal BA profile, as characterized by a reduced content of T $\alpha \mathrm{MCA}$, increased contents of TUDCA and LCA, together with increasing trends of GCDCA and HDCA contents. Several endogenous BAs can function as 
TABLE 6 | Effects of Clostridium butyricum treatment on ileal bile acids (BAs) ${ }^{1}$ contents of laying hens.

\begin{tabular}{|c|c|c|c|}
\hline & Control & Treatment & $P$-value \\
\hline \multicolumn{4}{|c|}{ Primary BAs (nmol/g) } \\
\hline $\mathrm{T} \alpha \mathrm{MCA}$ & $31.01 \pm 11.88^{a}$ & $17.58 \pm 6.24^{b}$ & 0.015 \\
\hline TßMCA & $3.68 \pm 1.58$ & $2.96 \pm 1.64$ & 0.426 \\
\hline GCA & $0.73 \pm 0.43$ & $1.07 \pm 0.47$ & 0.153 \\
\hline$\alpha \mathrm{MCA}$ & $5.24 \pm 4.64$ & $5.44 \pm 3.66$ & 0.926 \\
\hline$\beta M C A$ & $0.39 \pm 0.12$ & $0.38 \pm 0.20$ & 0.933 \\
\hline CDCA & $4520.76 \pm 821.24$ & $4836.78 \pm 1022.99$ & 0.456 \\
\hline CA & $496.84 \pm 215.04$ & $498.89 \pm 255.05$ & 0.986 \\
\hline GCDCA & $4.33 \pm 1.73$ & $6.65 \pm 2.90$ & 0.072 \\
\hline TCDCA & $3065.88 \pm 1571.45$ & $3923.87 \pm 2476.42$ & 0.454 \\
\hline \multicolumn{4}{|c|}{ Secondary BAs (nmol/g) } \\
\hline TUDCA & $0.69 \pm 0.44^{b}$ & $2.67 \pm 2.52^{\mathrm{a}}$ & 0.046 \\
\hline THDCA & $1.21 \pm 0.84$ & $0.57 \pm 0.31$ & 0.065 \\
\hline UCA & $0.66 \pm 0.34$ & $0.70 \pm 0.62$ & 0.885 \\
\hline UDCA & $5.00 \pm 1.81$ & $4.36 \pm 2.48$ & 0.555 \\
\hline $7-\mathrm{KDCA}$ & $0.39 \pm 0.16$ & $0.66 \pm 0.45$ & 0.130 \\
\hline 12-DHCA & $3.61 \pm 0.99$ & $4.11 \pm 2.16$ & 0.568 \\
\hline$\lambda \mathrm{MCA}$ & $57.03 \pm 42.35$ & $45.71 \pm 24.76$ & 0.553 \\
\hline 3-DHCA & $1.27 \pm 0.52$ & $1.88 \pm 0.92$ & 0.122 \\
\hline LCA & $0.31 \pm 0.12^{b}$ & $0.65 \pm 0.33^{a}$ & 0.016 \\
\hline 7-KLCA & $9.85 \pm 2.09^{b}$ & $17.46 \pm 7.32^{\mathrm{a}}$ & 0.009 \\
\hline 12-KLCA & $0.31 \pm 0.16$ & $0.40 \pm 0.15$ & 0.273 \\
\hline DCA & $0.56 \pm 0.22$ & $0.58 \pm 0.13$ & 0.763 \\
\hline ApCA & $9.60 \pm 3.21$ & $8.54 \pm 3.33$ & 0.502 \\
\hline AICA & $127.82 \pm 39.25$ & $114.41 \pm 42.87$ & 0.499 \\
\hline HDCA & $0.81 \pm 0.37$ & $1.17 \pm 0.37$ & 0.056 \\
\hline TLCA & $0.67 \pm 0.40$ & $0.86 \pm 0.38$ & 0.366 \\
\hline
\end{tabular}

a,b Values with different superscripts within the same row differ significantly $(P<0.05)$. ${ }^{1} T \alpha M C A$, tauro- $\alpha$-muricholic acid; T $\beta M C A$, tauro- $\beta$-muricholic acid; GCA, glycocholic acid; $\alpha M C A, \alpha$-muricholic acid; $\beta M C A, \beta$-muricholic acid; CDCA, chenodeoxycholic acid; CA, cholic acid; GCDCA, glycochenodeoxycholic acid; TCDCA, taurochenodeoxycholic acid; TUDCA, tauroursodeoxycholic acid; THDCA, taurohyodeoxycholic acid; UCA, ursocholic acid; UDCA, ursodeoxycholic acid; 7-KDCA, 7-ketodeoxycholic acid; 12-DHCA, 12-dehydrocholic acid; $\lambda M C A$, $\lambda$-muricholic acid; 3-DHCA, 3-dehydrocholic acid; $L C A$, lithocholic acid; 7-KLCA, 7-ketolithocholic acid; 12-KLCA, 12-ketolithocholic acid; DCA, deoxycholic acid; ApCA, apocholic acid; AICA, allocholic acid; HDCA, hyodeoxycholic acid; TLCA, taurolithocholic acid.

signaling molecules to interact with FXR, regulating not only their own synthesis and enterohepatic recirculation but also lipid metabolism (Rajani and Jia, 2018). Thereinto, TaMCA was identified as a FXR antagonist, whose reduction led to an elevated activity of FXR (Sayin et al., 2013). Conversely, LCA, GCDCA, TUDCA and HDCA were recognized as FXR agonists (Makishima et al., 2002; Marquardt et al., 2017; Wang et al., 2018). Remarkably, LCA and HDCA were found to be decreased in serum or intestine of animals with hepatic steatosis (Tang et al., 2019; Ushiroda et al., 2019), while dietary HDCA could exert hypolipidemic effects by reducing FXR antagonist BAs in enterohepatic tissues (Watanabe and Fujita, 2014). Accordingly, the current increases in TUDCA and LCA contents with the increasing trends of GCDCA and HDCA contents, as well as the reduced T $\alpha \mathrm{MCA}$ content in treatment group were naturally answerable to the corresponding elevation of ileal FXR expression. This could subsequently stimulate $\operatorname{PPAR} \alpha$-dependent $\beta$-oxidation of hepatic FAs through gut-liver crosstalk (Rajani and Jia, 2018), presumptively contributing to the reduced lipid deposition in the liver following C. butyricum addition. Because the majority of BAs are reabsorbed in the ileum into portal blood and re-circulated back to the liver for uptake by hepatocytes (Rajani and Jia, 2018; Tang et al., 2019), the elevated LCA, TUDCA, GCDCA and HDCA in the ileum induced by $C$. butyricum might transport to the liver via enterohepatic circulation and then directly activated hepatic FXR (as supported by the elevated expression of hepatic FXR in treatment group), thereby resulting in a repression of lipid deposition in the liver.

Biotransformation of BAs by gut microbiota through defined enzymatic activities such as deconjugation and dihydroxylation is a critical modulator of BA profile (Rajani and Jia, 2018; Ovadia et al., 2019). Associations between gut microbiota and the contents of various BAs have been previously confirmed (Tang et al., 2019; Ushiroda et al., 2019). Ovadia et al. (2019) observed a reduced conjugation of BAs in gut due to the increase of Bacteroidetes. Tang et al. (2019) indicated that Bacteroidetes favors HDCA formation probably by promoting deconjugation and dihydroxylation of primary BAs. Moreover, the abundances of Bacteroidetes together with Prevotellaceae were evidenced to be positively correlated with HDCA content, which might be the reason why their reductions contributes to obesity of host (Tang et al., 2019). Ushiroda et al. (2019) detected a positive correlation between Prevotellaceae abundance and LCA content. Herein, we found elevations in ileal LCA and HDCA contents concomitant with increased abundances of Bacteroidetes and Prevotellaceae in treatment group, suggesting that C. butyricum addition might promote LCA and HDCA production of layers by enriching Bacteroidetes and Prevotellaceae in gut. Clostridiales represents a typical producer of bile salt hydrolase, which catalyzes the deconjugation of conjugated BAs and promotes the formation of secondary BAs (Parasar et al., 2019). A positive relationship was identified between Clostridiales abundance in gut and deconjugation of taurocholic acid in animals (Ushiroda et al., 2019). Consequently, the increased abundance of Clostridiales following C. butyricum addition might promote the deconjugation of T $\alpha \mathrm{MCA}$, causing a corresponding decrease in ileal T $\alpha$ MCA content.

\section{CONCLUSION}

Supplemental C. butyricum accelerated FA oxidation and reduced lipid deposition in the liver of aged laying hens, which could be associated to the capability to shape gut microbial composition and BA profile that mediate the gut-liver crosstalk. The current study can expand our fundamental knowledge concerning the roles of gut microbiota in mediating the regulatory effect of $C$. butyricum on lipid metabolism, which certainly helps to improve the efficiency in applying C. butyricum in chicken production. 


\section{DATA AVAILABILITY STATEMENT}

The datasets generated for this study can be found in the Sequence Read Archive of the NCBI (Accession No. SAMN13610217).

\section{ETHICS STATEMENT}

The animal study was reviewed and approved by the Animal Care and Use Committee of the Feed Research Institute of the Chinese Academy of Agricultural Sciences.

\section{AUTHOR CONTRIBUTIONS}

WW performed the experiment and wrote the manuscript. JW and HZ assisted with data analysis. SW and GQ

\section{REFERENCES}

Boonsinchai, N. (2015). Effect of Feeding Regimen and Age on Lipid Metabolism in Broiler Breeder Hens and Progeny. Ph.D. dissertation, University of Arkansas, Fayetteville, AR.

Cani, P. D., Neyrinck, A. M., Fava, F., Knauf, C., Burcelin, R. G., Tuohy, K. M., et al. (2007). Selective increases of Bifidobacteria in gut microflora improve high-fat-diet-induced diabetes in mice through a mechanism associated with endotoxaemia. Diabetologia 50, 2374-2383. doi: 10.1007/s00125-007-0791-0

Carbajo-Pescador, S., Porras, D., Garcia-Mediavilla, M. V., Martinez-Florez, S., Juarez-Fernandez, M., Cuevas, M. J., et al. (2019). Beneficial effects of exercise on gut microbiota functionality and barrier integrity, and gut-liver crosstalk in an in vivo model of early obesity and non-alcoholic fatty liver disease. Dis. Mod. Mech. 12:dmm039206. doi: 10.1242/dmm.039206

Chu, C., Mao, L. F., and Schulz, H. (1994). Estimation of peroxisomal betaoxidation in rat heart by a direct assay of acyl-CoA oxidase. Biochem. J. 302, 23-29. doi: $10.1042 / b j 3020023$

Fang, S., Suh, J. M., Reilly, S. M., Yu, E., Osborn, O., Lackey, D., et al. (2015). Intestinal FXR agonism promotes adipose tissue browning and reduces obesity and insulin resistance. Nat. Med. 21, 71-77. doi: 10.1038/nm.3760

Folch, J., Lees, M., and Sloane-Stanley, G. (1957). A simple method for the isolation and purification of total lipids from animal tissues. J. Biol. Chem. 226, 497-509.

Fontaine, M. A., Diane, A., Singh, V. P., Mangat, R., Krysa, J. A., Nelson, R., et al. (2019). 4 Low birth weight causes insulin resistance and aberrant intestinal lipid metabolism independent of microbiota abundance in Landrace-Large White pigs. FASEB J. 33, 9250-9262. doi: 10.1096/fj.201801302rr

Fu, Z. D., Csanaky, I. L., and Klaassen, D. (2012). Gender-divergent profile of bile acid homeostasis during aging of mice. PLoS One 7:e32551. doi: 10.1371/ journal.pone.0032551

Griffin, M. J., and Sul, H. S. (2004). Insulin regulation of fatty acid synthase gene transcription: roles of USF and SREBP-1c. IUBMB Life 56, 595-600. doi: $10.1080 / 15216540400022474$

Hayashi, A., Sato, T., Kamada, N., Mikami, Y., Matsuoka, K., Hisamatsu, T., et al. (2013). A single strain of Clostridium butyricum induces intestinal IL10-producing macrophages to suppress acute experimental colitis in mice. Cell Host Microbe 13, 711-722. doi: 10.1016/j.chom.2013.05.013

Horton, J. D., Goldstein, J. L., and Brown, M. S. (2002). SREBPs: activators of the complete program of cholesterol and fatty acid synthesis in liver. J. Clin. Invest. 109, 1125-1131. doi: 10.1172/jci0215593

Jacobson, T. A., Glickstein, S. B., Rowe, J. D., and Soni, P. N. (2012). Effects of eicosapentaenoic acid and docosahexaenoic acid on low-density lipoprotein cholesterol and other lipids: a review. J. Clin. Lipidol. 6, 5-18. doi: 10.1016/j. jacl.2011.10.018

Jia, L. L., Li, D. Y., Feng, N. H., Shamoon, M., Sun, Z. H., Ding, L., et al. (2017). Antidiabetic effects of Clostridium butyricum CGMCC0313.1 through promoting contributed to the experimental design and preparation of the manuscript. All authors discussed the results and reviewed the manuscript and read and approved the final manuscript.

\section{FUNDING}

This work was financially supported by the National Key R\&D Program of China (2018YFD0500600) and the China Postdoctoral Science Foundation (2019M650924).

\section{SUPPLEMENTARY MATERIAL}

The Supplementary Material for this article can be found online at: https://www.frontiersin.org/articles/10.3389/fmicb. 2020.00600/full\#supplementary-material

the growth of gut butyrate-producing bacteria in type 2 diabetic mice. Sci. Rep. 7:7046.

Keskitalo, A., Munukka, E., Toivonen, R., Hollmen, M., Kainulainen, H., Huovinen, P., et al. (2018). Enterobacter cloacae administration induces hepatic damage and subcutaneous fat accumulation in high-fat diet fed mice. PLoS One 13:e0198262. doi: 10.1371/journal.pone.0198262

Knottnerus, S. J. G., Bleeker, J. C., Wust, R. C. I., Ferdinandusse, S., IJlst, L., Wijburg, F. A., et al. (2018). Disorders of mitochondrial long-chain fatty acid oxidation and the carnitine shuttle. Rev. Endocr. Metab. Dis. 19, 93-106. doi: 10.1007/s11154-018-9448-1

Li, F., Shan, M. X., Gao, X., Yang, Y., Yang, X., Zhang, X. Y., et al. (2019). Effects of nutrition restriction of fat- and lean-line broiler breeder hens during the laying period on offspring performance, blood biochemical parameters, and hormone levels. Domest. Anim. Endocrin. 68, 73-82. doi: 10.1016/j.domaniend.2019.0 1.007

Liu, J. M., Fu, Y. Y., Zhang, H., Wang, J. D., Zhu, J., Wang, Y. Q., et al. (2017). The hepatoprotective effect of the probiotic Clostridium butyricum against carbon tetrachloride-induced acute liver damage in mice. Food Funct. 8, 4042-4052. doi: 10.1039/c7fo00355b

Liu, X. T., Lin, X., Mi, L. Y., Zeng, W. D., and Zhang, C. Q. (2018). Age-related changes of yolk precursor formation in the liver of laying hens. J. Zhejiang Univ-Sci. B Biomed. Biotechnol. 19, 390-399. doi: 10.1631/jzus.B1700054

Lu, M. W., Cao, Y., Xiao, J., Song, M. Y., and Ho, C. T. (2018). Molecular mechanisms of the anti-obesity effect of bioactive ingredients in common spices: a review. Food Funct. 9, 4569-4581. doi: 10.1039/c8fo01349g

Lu, X. J., Liu, J. X., Zhang, N. S., Fu, Y. H., Zhang, Z. C., Li, Y. X., et al. (2019). Ripened Pu-erh tea extract protects mice from obesity by modulating gut microbiota composition. J. Agric. Food Chem. 67, 6978-6994. doi: 10.1021/acs. jafc.8b04909

Makishima, M., Lu, T. T., Xie, W., Whitfield, G. K., Domoto, H., Evans, R. M., et al. (2002). Vitamin D receptor as an intestinal bile acid sensor. Science 296, 1313-1316. doi: 10.1126/science.1070477

Marquardt, A., Al-Dabet, M. M., Ghosh, S., Kohli, S., Manoharan, J., ElWakiel, A., et al. (2017). Farnesoid X receptor agonism protects against diabetic tubulopathy: potential add-on therapy for diabetic nephropathy. J. Am. Soc. Nephrol. 28, 3182-3189. doi: 10.1681/ASN.2016101123

Mirza, R., and Sharma, B. (2019). A selective peroxisome proliferatoractivated receptor-gamma agonist benefited propionic acid induced autismlike behavioral phenotypes in rats by attenuation of neuroinflammation and oxidative stress. Chem. Biol. Interact. 311:108758. doi: 10.1016/j.cbi.2019. 108758

Monk, J. M., Wu, W. Q., Lepp, D., Wellings, H. R., Hutchinson, A. L., Liddle, D. M., et al. (2019). Navy bean supplemented high-fat diet improves intestinal health, epithelial barrier integrity and critical aspects of the obese inflammatory phenotype. J. Nutr. Biochem. 70, 91-104. doi: 10.1016/j.jnutbio.2019.04.009 
Mossab, A., Lessire, M., Guillaumin, S., Kouba, M., Mourot, J., Peiniau, P., et al. (2002). Effect of dietary fats on hepatic lipid metabolism in the growing turkey. Comp. Biochem. Physiol. B Biochem. Mol. Biol. 132, 473-483. doi: 10.1016/ s1096-4959(02)00059-3

Mulvihill, E. E. (2018). Regulation of intestinal lipid and lipoprotein metabolism by the proglucagon-derived peptides glucagon like peptide 1 and glucagon like peptide 2. Curr. Opin. Lipidol. 29, 95-103. doi: 10.1097/MOL. 0000000000000495

Murugesan, S., Nirmalkar, K., Hoyo-Vadillo, C., García-Espitia, M., RamírezSánchez, D., and García-Mena, J. (2018). Gut microbiome production of shortchain fatty acids and obesity in children. Eur. J. Clin. Microbiol. 37, 621-625. doi: 10.1007/s10096-017-3143-0

National Research Council [NRC], (1994). Nutrient Requirements of Poultry: Ninth Revised Edition. Washington, DC: The National Academies Press.

Ovadia, C., Perdones-Montero, A., Spagou, K., Smith, A., Sarafian, M. H., Gomez-Romero, M., et al. (2019). Enhanced microbial bile acid deconjugation and impaired ileal uptake in pregnancy repress intestinal regulation of bile acid synthesis. Hepatology 70, 276-293. doi: 10.1002/hep. 30661

Parasar, B., Zhou, H., Xiao, X. Y., Shi, Q. J., Brito, I. L., and Chang, P. V. (2019). Chemoproteomic profiling of gut microbiota-associated bile salt hydrolase activity. ACS Cent. Sci. 5, 867-873. doi: 10.1021/acscentsci.9b00147

Pope, J. L., Yang, Y., Newsome, R. C., Sun, W., Sun, X. L., Ukhanova, M., et al. (2019). Microbial colonization coordinates the pathogenesis of a Klebsiella pneumoniae infant isolate. Sci. Rep. 9:3380. doi: 10.1038/s41598-019-39887-8

Rajani, C., and Jia, W. (2018). Bile acids and their effects on diabetes. Front. Med. 12:608-623. doi: 10.1007/s11684-018-0644-x

Ringseis, R., and Eder, K. (2009). Influence of pharmacological PPARalpha activators on carnitine homeostasis in proliferating and non-proliferating species. Pharmacol. Res. 60, 179-184. doi: 10.1016/j.phrs.2009. 01.016

Saleh, A. A., Eid, Y. Z., Ebeid, T. A., Ohtsuka, A., Hioki, K., Yamamoto, M., et al. (2012). The modification of the muscle fatty acid profile by dietary supplementation with Aspergillus awamori in broiler chickens. Br. J. Nutr. 108, 1596-1602. doi: 10.1017/S0007114511007069

Saleh, A. A., Hayashi, K., Ijiri, D., and Ohtsuka, A. (2014). Beneficial effects of Aspergillus awamori in broiler nutrition. World Poult. Sci. J. 70, 857-864. doi: 10.1017/s0043933914000907

Sato, R., and Tanaka, M. (1997). Intestinal distribution and intraluminal localization of orally administered Clostridium butyricum in rats. Microbiol. Immunol. 41, 665-671. doi: 10.1111/j.1348-0421.1997.tb01909.x

Sato, S., Nagai, H., and Igarashi, Y. (2012). Effect of probiotics on serum bile acids in patients with ulcerative colitis. Hepato Gastroenterol. 59, 1804-1808.

Sayin, S. I., Wahlstrom, A., Felin, J., Jantti, S., Marschall, H. U., Bamberg, K., et al. (2013). Gut microbiota regulates bile acid metabolism by reducing the levels of tauro-beta-muricholic acid, a naturally occurring FXR antagonist. Cell Metab. 17, 225-235. doi: 10.1016/j.cmet.2013.01.003

Schloss, P. D., Westcott, S. L., Ryabin, T., Hall, J. R., Hartmann, M., Hollister, E. B., et al. (2009). Introducing mothur: open-source, platform-independent, community supported software for describing and comparing microbial communities. Appl. Environ. Microbiol. 75, 7537-7541. doi: 10.1128/AEM. 01541-09

Seo, M., Inoue, I., Tanaka, M., Matsuda, N., Nakano, T., Awata, T., et al. (2013). Clostridium butyricum MIYAIRI 588 improves high-fat diet-induced nonalcoholic fatty liver disease in rats. Dig. Dis. Sci. 58, 3534-3544. doi: 10.1007/ s10620-013-2879-3

Shang, H. X., Sun, J., and Chen, Y. Q. (2016). Clostridium butyricum CGMCC0313.1 modulates lipid profile, insulin resistance and colon homeostasis in obese mice. PLoS One 11:e0154373. doi: 10.1371/journal. pone. 0154373

Sinal, C. J., Tohkin, M., Miyata, M., Ward, J. M., Lambert, G., and Gonzalez, F. J. (2000). Targeted disruption of the nuclear receptor FXR/BAR impairs bile acid and lipid homeostasis. Cell 102, 731-744. doi: 10.1016/s0092-8674(00)00062-3

Tang, Y. Y., Zhang, J. Y., Li, J., Lei, X. H., Xu, D. K., Wang, Y., et al. (2019). Turnover of bile acids in liver, serum and caecal content by high-fat diet feeding affects hepatic steatosis in rats. BBA-Mo. Cell Biol. L 1864, 1293-1304. doi: 10.1016/j.bbalip.2019.05.016
Trott, K. A., Giannitti, F., Rimoldi, G., Hill, A., Woods, L., Barr, B., et al. (2014). Fatty liver hemorrhagic syndrome in the backyard chicken. Vet. Pathol. 51, 787-795. doi: 10.1177/0300985813503569

Ushiroda, C., Naito, Y., Takagi, T., Matsuda, N., Nakano, T., Awata, T., et al. (2019). Green tea polyphenol (epigallocatechin-3-gallate) improves gut dysbiosis and serum bile acids dysregulation in high-fat diet-fed mice. J. Clin. Biochem. Nutr. 65, 34-46. doi: 10.3164/jcbn.18-116

Videnska, P., Sedlar, K., Lukac, M., Faldynova, M., Gerzova, L., Cejkova, D., et al. (2014). Succession and replacement of bacterial populations in the caecum of egg laying hens over their whole life. PLoS One 9:e115142. doi: 10.1371/journal. pone.0115142

Wang, B., and Tontonoz, P. (2018). Liver X receptors in lipid signalling and membrane homeostasis. Nat. Rev. Endocrinol. 14, 452-463. doi: 10.1038/ s41574-018-0037-x

Wang, F., Lu, Z., Wang, X., and Zhang, Y. (2018). Impaired vagus function in rats suppresses bile acid synthesis in liver by disrupting tight junctions and activating Fxr-Fgf15 signaling in the intestine. Biochem. Biophys. Res. Commun. 495, 1490-1496. doi: 10.1016/j.bbrc.2017.11.201

Wang, J., Tang, H., Zhang, C., Zhao, Y., Derrien, M., Rocher, E., et al. (2015). Modulation of gut microbiota during probiotic-mediated attenuation of metabolic syndrome in high fat diet-fed mice. ISME J. 9, 1-15. doi: 10.1038/ ismej.2014.99

Wang, W. W., Jia, H. J., Zhang, H. J., Wang, J., Lv, H. Y., Wu, S. G., et al. (2019). Supplemental plant extracts from Flos lonicerae in combination with Baikal skullcap attenuate intestinal disruption and modulate gut microbiota in lying hens challenged by Salmonella pullorum. Front. Microbiol. 10:1681. doi: $10.3389 /$ fmicb.2019.01681

Watanabe, S., and Fujita, K. (2014). Dietary hyodeoxycholic acid exerts hypolipidemic effects by reducing farnesoid $\mathrm{X}$ receptor antagonist bile acids in mouse enterohepatic tissues. Lipids 49, 963-973. doi: 10.1007/s11745-0143947-y

Weng, H., Endo, K., Li, J., Kito, N., and Iwai, N. (2015). Induction of peroxisomes by butyrate-producing probiotics. PLoS One 10:e0117851. doi: 10.1371/journal. pone.0117851

Yoo, S. R., Kim, Y. J., Park, D. Y., Jung, U. J., Jeon, S. M., Ahn, Y. T., et al. (2013). Probiotics L. plantarum and L. curvatus in combination alter hepatic lipid metabolism and suppress diet-induced obesity. Obesity 21:12.

Zhang, B. K., Yang, X. J., Guo, Y. M., and Long, F. Y. (2011). Effects of dietary lipids and Clostridium butyricum on serum lipids and lipid-related gene expression in broiler chickens. Animal 5, 1909-1915. doi: 10.1017/s1751731111001066

Zhang, C. C., Wu, W. C., Li, X. Q., Xin, X. T., and Liu, D. Q. (2019). Daily supplementation with fresh Angelica keiskei juice alleviates high-fat dietinduced obesity in mice by modulating gut microbiota composition. Mol. Nutr. Food Res. 63:1900248. doi: 10.1002/mnfr.201900248

Zhang, L., Cao, G. T., Zeng, X. F., Zhou, L., Ferket, P. R., Xiao, Y. P., et al. (2014). Effects of Clostridium butyricum on growth performance, immune function, and cecal microflora in broiler chickens challenged with Escherichia coli K88. Poult. Sci. 93, 46-53. doi: 10.3382/ps.2013-03412

Zhao, E. Z., Khafipour, E., Sepehri, S., Huang, F., Beta, T., and Shen, G. X. (2019). Impact of Saskatoon berry powder on insulin resistance and relationship with intestinal microbiota in high fat-high sucrose diet-induced obese mice. J. Nutr. Biochem. 69, 130-138. doi: 10.1016/j.jnutbio.2019.03.023

Zhao, X., Guo, Y. M., Guo, S. S., and Tan, J. Z. (2013). Effects of Clostridium butyricum and Enterococcus faecium on growth performance, lipid metabolism, and cecal microbiota of broiler chickens. Appl. Microbiol. Biotechnol. 97, 64776488. doi: 10.1007/s00253-013-4970-2

Conflict of Interest: The authors declare that the research was conducted in the absence of any commercial or financial relationships that could be construed as a potential conflict of interest.

Copyright (C) 2020 Wang, Wang, Zhang, Wu and Qi. This is an open-access article distributed under the terms of the Creative Commons Attribution License (CC BY). The use, distribution or reproduction in other forums is permitted, provided the original author(s) and the copyright owner(s) are credited and that the original publication in this journal is cited, in accordance with accepted academic practice. No use, distribution or reproduction is permitted which does not comply with these terms. 